\title{
Uncertainty-limited turnstile transport in deformed microcavities
}

\author{
Jeong-Bo Shim, ${ }^{1,2}$ Sang-Bum Lee, ${ }^{1}$ Sang Wook Kim, ${ }^{3}$ Soo-Young Lee, ${ }^{1}$ \\ Juhee Yang, ${ }^{1}$ Songky Moon, ${ }^{1}$ Jai-Hyung Lee, ${ }^{1}$ and Kyungwon $\mathrm{An}^{1, *}$ \\ ${ }^{1}$ School of Physics and Astronomy, Seoul National University, Seoul, 151-742, Korea \\ ${ }^{2}$ Max-Planck Institute for Physics of Complex Systems, Nöthnitzer Str. 38, Dresden, Germany \\ ${ }^{3}$ Department of Physics Education, Pusan National University, Busan 609-735, Korea
}

(Dated: January 7, 2008)

\begin{abstract}
We present both experimental and theoretical evidences for uncertainty-limited turnstile transport in deformed microcavities. As the degree of cavity deformation was increased, a secondary peak gradually emerged in the far-field emission patterns to form a double-peak structure. Our observation can be explained in terms of the interplay between turnstile transport and its suppression by the quantum mechanical uncertainty principle.
\end{abstract}

PACS numbers: 05.45.Mt, 42.55.Sa, 73.24.-b

In the studies of quantum counterparts of classical chaotic systems, we often encounter a situation in which classical diffusion is suppressed in quantum mechanics by the intrinsic limitation of the resolvable action quantity [1] due to the Heisenberg uncertainty principle. Partial barrier localization [2-5] and the suppression of multiphoton ionization [6] are well-known examples. This suppression phenomenon would become more conspicuous when a Hamiltonian system takes a gradual transition to chaos so that the action transport by chaotic dynamics also increases along the chaotic transition $[7,8]$.

Recently, many works have converged to a consensus that the emission directionality in chaotic deformed microcavities is well explained by classical ray dynamics in phase space [9-16]. However, the evanescent leakage from a symmetric or slightly deformed microcavity is inexplicable by the classical dynamics [17], and thus it is of considerable interest to understand how emission mechanism changes along the chaotic transition. In this context we can expect that the resolvability of action quantity would also play an important role in light transport and thus emission directionality in deformed microcavities. Such understanding is important not only for theoretical interests, but also for the practical purpose of optimizing the directional emission with a high cavity quality factor $Q$ for various photonics applications.

In this letter, we elucidate the effect of action resolvability on the light transport in a deformed microcavity with continuously variable shape deformation. We observed in both experiment and theory that output emission is characterized by the emergence of a sharp secondary peak as the degree of cavity deformation is increased. These double peaks originate from two separate phase-space lobes in the so-called turnstile transport $[18,19]$ for whispering-gallery-mode-like quasieigenmodes. Moreover, the gradual emergence of the secondary peak is a direct evidence for suppression of chaotic diffusion due to the quantum mechanical uncertainty principle.

Our deformed microcavity is realized by optically ex-

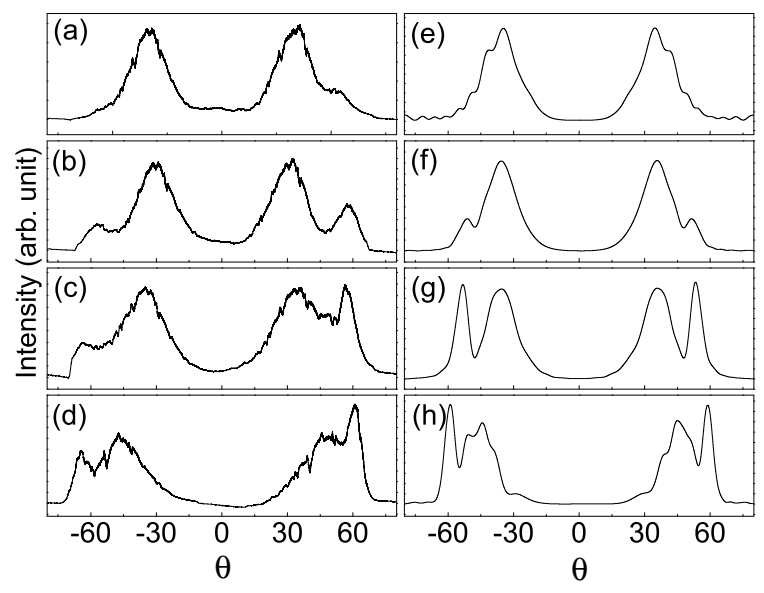

FIG. 1: (a)-(d) Far-field emission patterns observed in experiment and (e)-(h) those in wave calculation. Degree of cavity deformation $\eta$ is 0.09 for (a) and (e), 0.10 for (b) and (f), 0.12 for (c) and (g) and 0.16 for (d) and (h). As the chaotic transition progresses, a secondary narrow peak emerges around $\theta \sim \pm 55^{\circ}$ in the presence of a broad peak around $\theta \sim \pm 40^{\circ}$. The baseline by the horizontal axis corresponds to zero signal. All plots are normalized.

citing a thin cross-sectional volume across a liquid jet column of ethanol (refractive index $m=1.361$ ) doped with Rhodamine $\mathrm{B}$ dye at a concentration of $10^{-7} \mathrm{~mol} / \mathrm{cm}^{3}$. The details of our liquid-jet apparatus is described elsewhere [20]. Surface profiling measurement based on forward shadow diffraction shows that the shape of our cavity is described by $r(\phi)=a\left(1+\eta_{0} \cos 2 \phi+\epsilon \eta_{0}^{2} \cos 4 \phi\right)$ in the polar coordinate with $\eta_{0}$ continuously variable from 0 to $26 \%$ with $\epsilon \simeq 0.46 \pm 0.05$ and $a \simeq 14.8 \mu \mathrm{m}$. The ray dynamics in this shape becomes chaotic following the Kolmogorov-Arnold-Moser (KAM) scenario as $\eta_{0}$ increases. Accordingly, it can be approximated by another KAM system, the quadrupolar billiard [21], the boundary of which is given by $r(\phi)=a(1+\eta \cos 2 \phi)$ while 


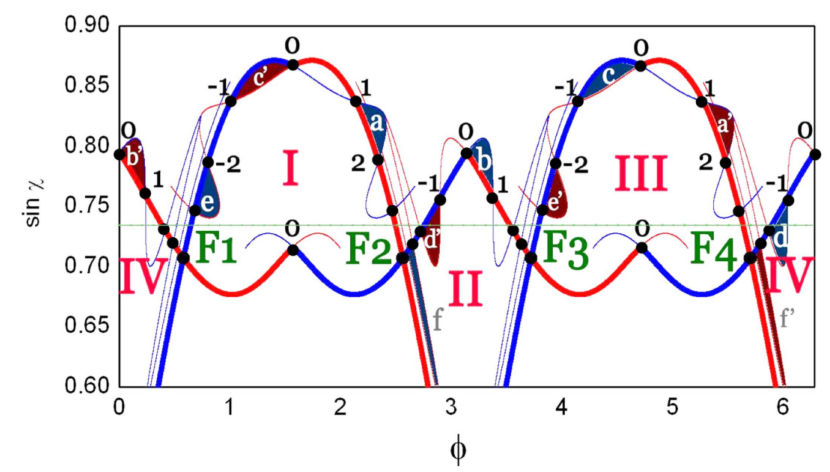

FIG. 2: Phase-space structure of stable (blue) and unstable (red) manifolds of the unstable period- 4 orbit $F_{n}(n=1,2,3$, 4) for $\eta=0.12$, presented in Birkhoff's coordinates, in which a ray collides with the cavity boundary at polar angle $\phi$ with an incidence angle $\chi$. Chaotic transport from the region above the chained areas into the chained areas (I, II, III, IV) occurs by the lobes dynamics $\mathbf{a} \rightarrow \mathbf{b} \rightarrow \mathbf{c} \rightarrow \mathbf{d} \rightarrow \mathbf{e} \rightarrow \mathbf{f}$, the order of which is given by the heteroclinic orbit points $(\ldots,-2,-1,0,1,2)$.

the overall structures of their phase spaces are very similar to each other except for the chaotic transition speed: $\eta \simeq \eta_{0}-(0.05 \sim 0.08)$. For the convenience of analysis, our numerical works have been performed for the equivalent quadrupolar deformed microcavity.

The farfield emission patterns of lasing modes observed in our experiment is shown in Fig. 1, compared with the calculated emission patterns based on the boundary element method [22, 23] for various cavity deformations, covering from the mixed-phase-space regime $(\eta=0.09)$ to the fully chaotic one $(\eta=0.16)$. The experimental procedure for measuring the far-field emission patterns is similar to that of Ref. [14]. In the wave calculation, the size of the cavity is characterized by the so-called size parameter $x=2 \pi m a / \lambda$ with $\lambda$ the wavelength. In our case, $x$ is in the range of 220 . The most noticeable feature in both experiment and wave calculation is the emergence of a narrow secondary peak around $\theta= \pm\left(55^{\circ} \sim 60^{\circ}\right)$ as the cavity deformation increases.

In order to understand this double-peak structure, let us first consider the manifold structure, especially given by the unstable period- 4 orbit. The reason for considering the period-4 orbit only is that the ray dynamics in relevant phase space region is mainly governed by the period-4 orbit since it is the shortest periodic orbit near the line of critical angle. Figure 2 presents the phase space diagram in the Birkhoff's coordinate [24] for the quadrupolar billiard. In this diagram the fixed points of the period- 4 orbit are indexed by $F_{n}(n=1,2,3,4)$. A stable manifold (in blue) and an unstable manifold (in red) from each fixed point constitute a heteroclinic tangled structure, where the central intersecting points, marked by 0's, of two opposite types of manifolds are set as primary intersection points (PIP's).

The segments of manifolds connecting the PIP's en-
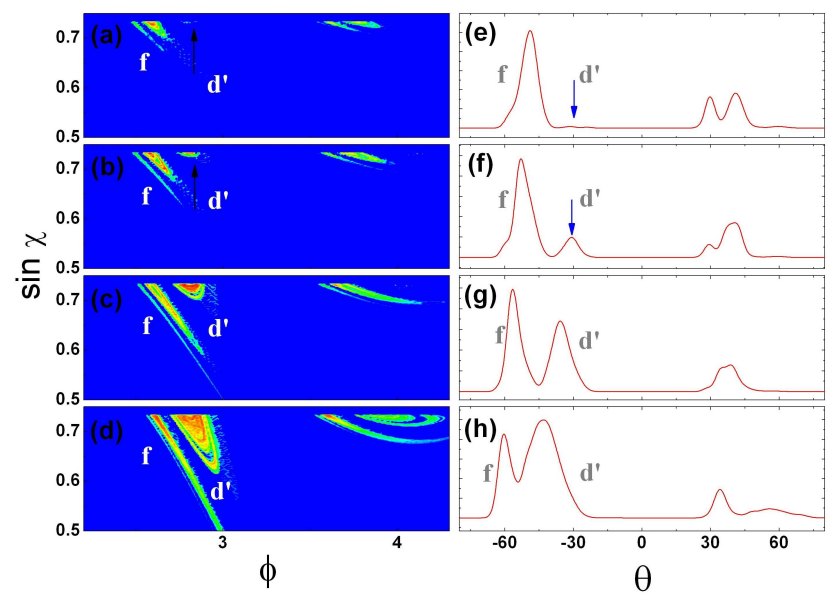

FIG. 3: Results of ray tracing simulations for (a) $\eta=0.09$, (b) 0.10, (c) 0.12 and (d) 0.16. In (a)-(d) it is shown that lobe d' comes down below the critical line while the already existing lobe $\mathbf{f}$ moves further downwards. Plots in (e)-(h) show the corresponding output angular distributions histogramatically calculated in the ray simulation. A narrow peak around $55^{\circ}$ originating from lobe $\mathbf{f}$ is ever present while a broad peak from lobe d' gradually grows as $\eta$ increases.

close four chained areas marked by I, II, III and IV. The quasieigenmodes with considerably high $Q$ for lasing should be distributed in the phase space well above the chained areas [16]. It is because the chained areas I and III are respectively connected to II and IV, whose significant portions are placed below the critical line, by a few iterations of ray reflection off the boundary. Therefore, any mode with its distribution substantially enclosed in the chained areas tends to experience large losses.

According to the chaotic transport theory [18, 19], however, the region above the chained areas can be transported to the chained areas through lobes (marked by a, $\mathbf{b}, \mathbf{c}, \ldots)$, small subareas enclosed by the segments of the stable and unstable manifolds connecting the heteroclinic orbit points indexed by $\ldots-2,-1,0,1,2, \ldots$ in Fig. 2. In this theory, lobes are transported in the sequence of $\mathbf{a} \rightarrow \mathbf{b} \rightarrow \mathbf{c} \rightarrow \mathbf{d} \rightarrow \mathbf{e} \rightarrow \mathbf{f} .$. and the region enclosed by a lobe above the chained areas can be transported to the chained areas when the sequence crosses a PIP. Such a transport across a manifold barrier is called turnstile transport. In Fig. 2, lobes $\mathbf{d}$ and $\mathbf{f}$, whose significant portions lie below the critical line, then form two separate output channels. We can also find the similar sequence of another lobe dynamics $\mathbf{a}^{\prime} \rightarrow \mathbf{b}^{\prime} \rightarrow \mathbf{c}^{\prime} \rightarrow \mathbf{d}^{\prime} \rightarrow \mathbf{e}^{\prime} \rightarrow \mathbf{f}^{\prime} \ldots$, which also lead to two additional output channels, lobes $\mathbf{d}$ ' and $\mathbf{f}^{\prime}$, which are $180^{\circ}$ shifted from lobes $\mathbf{d}$ and $\mathbf{f}$, respectively.

Combining these two lobe dynamics, we can account for the double-peak structure in the far-field emission patterns. For this end, we performed a ray-tracing simulation with $10^{6}$ counterclockwise rays (seen from the $z$ direction in the polar coordinate) uniformly prepared in the region above the chained areas initially. The output 
intensity is calculated for each ray by using the Fresnel formula whenever it is reflected with its incident angle smaller than the critical angle. The output intensity is accumulated histogramatically on the phase space, resulting in the phase-space plots in Figs. 3(a)-3(d). The output angular distributions in Figs. 3(e)-3(h) are similarly obtained by accumulating the output intensity histogramatically as a function of the refraction angle.

The results in Fig. 3 clearly show that lobe d' and lobe f of the turnstile process form two separate output channels. The output angular distribution in Figs. 3(e)-3(h) resemble the far-field emission patterns observed in the experiment and in the wave calculation. We can identify that the broad peaks in Fig. 1 correspond to lobe d' while the narrow secondary peaks in Fig. 1 correspond to lobe f.

However, there exists a significant discrepancy between the experiment/wave calculation and the ray simulation in the relative sizes of these two peaks. In the experiment/wave calculation, the primary broad peak is ever present while the narrow secondary peak gradually emerges as $\eta$ increase. On the other hand, in the ray simulation, the secondary peak is already there while the primary peak grows as $\eta$ increases. Note that only the left half of the output angular distribution is obtained in the ray simulation since only the counterclockwise rays are considered.

It will be shown below that this discrepancy is due to the quantum mechanical effect associated with the finite sizes of lobes and partial barriers in the phase space. If the output emission entirely came from the turnstile ray transport, the primary peak observed in the experiment/wave calculation should not be there according to the ray simulation result of Fig. 3(e). In addition, in the experiment/wave calculation of Fig. 1 (a) with $\eta=0.09$, we only see a hint of the secondary peak, which must come from the turnstile transport according to Fig. 3(e). Except that small contribution, the rest of the distribution should come from a different emission mechanism.

We show that this mechanism is quantum mechanical tunneling. In the mixed phase-space regime of $\eta=0.09$, the lowermost boundary of whispering-gallery-like high$Q$ modes are distributed just below the period-6 orbit. At this cavity deformation, all KAM tori in the region between the period- 6 orbit and the period- 4 orbit are broken, but some of them still remain as Cantor's sets or cantori forming partial barriers. The action transport through the cantori is allowed classically, but in quantum mechanics or wave mechanics it is allowed only when its action quantity is larger than a half of an effective Planck constant $\hbar_{\text {eff }}$, the quantum mechanical resolution limit in the phase space. In our system, the effective Planck constant is given by the inverse of the size parameter $x$ : $\hbar_{\text {eff }}=1 / x \sim 0.005$.

When $\eta=0.09$, the action quantity associated with the cantori is found to be much less than $\hbar_{\mathrm{eff}} / 2$. Therefore,
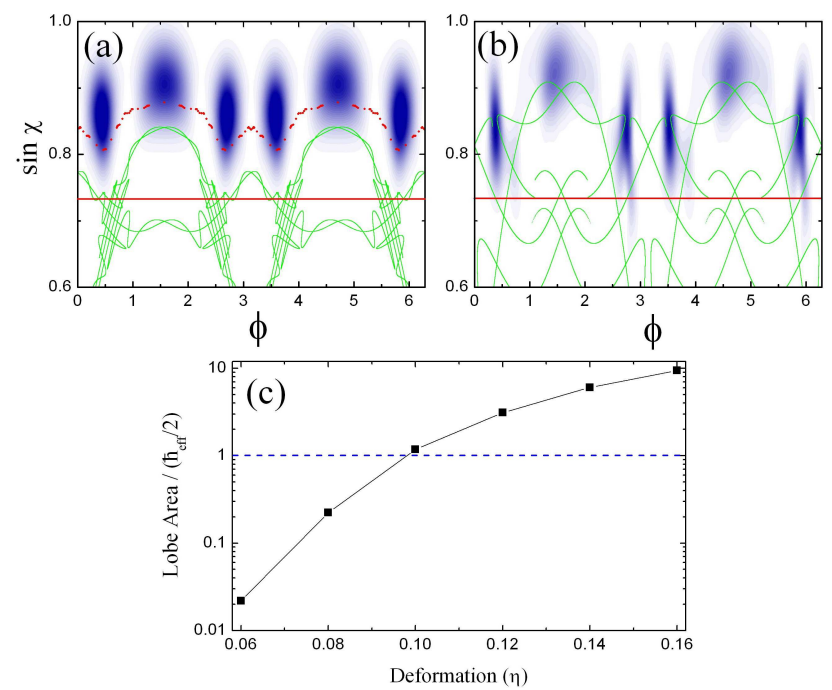

FIG. 4: Husimi distribution (blue) for (a) $\eta=0.09$ and (b) $\eta=0.16$. The heteroclinic tangled structure of the period- 4 oribit are represented as green curves. The horizontal thick red line is the line of critical angle. In (a) the cantori represented as red dots blocks the distribution from overlapping with the tangled structure. In (b) the distribution has a considerable overlap with the manifold structure. (c) The ratio of the lobe area to the resolution limit $\hbar_{\mathrm{eff}} / 2$ as a function of the deformation parameter. The blue dotted line represents the resolution limit under our experimental condition. The lobe area becomes comparable to $\hbar_{\text {eff }} / 2$ when $\eta \sim 0.1$.

the action transport through the cantori is greatly suppressed. In this case, the only output mechanism for the high- $Q$ modes is the evanescent tunneling through the closest point between the cantori and the critical line as shown in Fig. 4(a), where Husimi distribution for a relevant high- $Q$ mode is overlapped with the phase space structure. The angular position of the primary peaks in Figs. 1(a) and 1(e) coincides with the location of tunneling in Fig. 4(a), supporting this reasoning.

According to Fig. 4(a), the Husimi distribution also overlaps with manifold structure with the same order of magnitude as it does with the critical line. The distribution overlapped with lobes c' and a, for example, can then be transported to lobes $\mathbf{d}$ ' and $\mathbf{f}$. Therefore, we expect to see an equally strong secondary peak in the far-field distribution in Fig. 1. Instead, we only see a hint of the secondary peak at most. It is because the action quantity associated with lobes are also much smaller than the quantum resolution limit, $\hbar_{\mathrm{eff}} / 2$, and thus the turnstile transport is greatly suppressed.

In generic chaotic systems, the lobe areas increase as the transition to chaos progresses. Figure 4(c) shows the calculated action quantity associated with the lobes around the period- 4 orbit. The action quantity rapidly grows as $\eta$ increases and surpasses the resolution limit $\hbar_{\text {eff }} / 2 \sim 0.0025$ around $\eta=0.10$. In the mixed phasespace regime of $\eta=0.09$, the action quantity is still smaller 

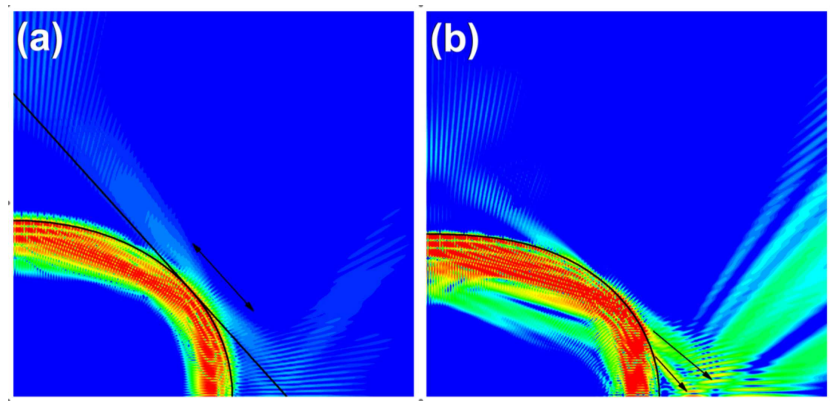

FIG. 5: Configurational plots of quasi-eigenmodes. (a) $\eta=0.09$ and $x=221.07-0.005 \mathrm{i}, \quad(\mathrm{b}) \quad \eta=0.16$ and $x=227.65-$ 0.024i. In (a) the emission, coming from evanescent leakage, has its direction tangent to the boundary. In (b) the emission comes from turnstile transport, and thus it is asymmetric and refractive. Dual strong emissions to $\theta \sim-45^{\circ},-60^{\circ}$ directions are visible.

than the resolution limit. Therefore, the high- $Q$ modes, although their distributions overlap with the lobe structure to some degree, cannot recognize the presence of these lobes, and thus the turnstile transport is greatly suppressed. This is why the secondary peak is barely seen in the far-field emission pattern in the experiment and wave calculation for $\eta=0.09$. In this case, the output is made by the evanescent tunneling, and consequently its emission direction is tangent to the cavity boundary at the tunneling points with its angular divergence given by the Fresnel filtering effect [25] as shown in Fig. 5(a), the spatial mode distribution by wave calculation.

In the same line of reasoning, the growth of the secondary peak in the experiment/wave calculation can be understood. As the degree of cavity deformation increases beyond $\eta=0.1$, the action quantities associated with the cantori and the lobes become larger than $h_{\mathrm{eff}} / 2$, and thus the action transport through the cantori and consequently the turnstile transport through the lobes become dominant mechanisms for the output emission. Accordingly, the portion of the output due to the evanescent tunneling is reduced. In the full chaotic regime such as in $\eta=0.16$, the Husimi distribution corresponding to a scarred mode $[10,14,16,25,26]$ shows considerable overlap with the manifold structure as shown in Fig. 4(b), and thus most of the output emission is due to the action transport. This is confirmed by the same emission patterns observed in both experiment/wave calculation and the ray simulation. The most prominent feature of this mechanism is the double-peak emission with each peak coming from a separate lobe. In this case, the emission is refractive in nature as can be seen in Fig. 5(b).

In conclusion, we have observed the turnstile light transport and its suppression due to the action resolvability in deformed microcavities. Our findings can explain the observed robustness of high $Q$ modes in chaotic microcavities [21, 26]; light can be strongly confined above the critical line due to this suppression effect even in the chaotic phase space. We expect our results can also be applicable to other open nonintegrable systems such as quantum dots and nano structures, where efficient control of electron transport for device applications might be possible via manipulation of the effective Planck constant.

* Electronic address: kwan@phya.snu.ac.kr

[1] L. E. Reichl, The Transition to Chaos in Conservative Classical Systems: Quantum Manifestations (SpringerVerlag, New York, 2004).

[2] R. C. Brown and R. E. Wyatt, Phys. Rev. Lett. 57, 1 (1986).

[3] T. Geisel, G. Radon, and J. Rubner, Phys. Rev. Lett. 57, 2883 (1986).

[4] K. Vant, G. Ball, H. Ammann, and N. Christensen, Phys. Rev. E 59, 2846 (1999)

[5] S. W. Kim, and H.-W. Lee, Phys. Rev. E 61, 5124 (2000).

[6] R. S. MacKay, and J. D. Meiss, Phys. Rev. A 37, 4702 (1988).

[7] G. Casati and T. Prosen, Phys. Rev. E 59 2516(R) (1999).

[8] N. T. Maitra and E. J. Heller, Phys. Rev. E 613620 (2000).

[9] H. G. L. Schwefel, N. B. Rex, H. E. Tureci, R. K. Chang, and A. D. Stone, J. Opt. Soc. Am. B 21, 923 (2004).

[10] S.-Y. Lee, J.-W. Ryu, T.-Y. Kwon, S. Rim, and C.-M. Kim, Phys. Rev. A 72, 061801(R) (2005).

[11] J. Wiersig and M. Hentschel, Phys. Rev. A 73 031802(R) (2006).

[12] R. Schäfer, U. Kuhl, and H.-J. Stöckmann, New J. Phys. 8, 46 (2006).

[13] S. Shinohara, and T. Harayama, Phys. Rev. E 75, 036216 (2007).

[14] S.-B. Lee, J. Yang, S. Moon, J.-H. Lee, K. An, J.-B. Shim, H.-W. Lee, and S. W. Kim, Phys. Rev. A 75, 011802(R) (2007)

[15] T. Tanaka, M. Hentschel, T. Fukushima, and T. Harayama, Phys. Rev. Lett. 98, 033902 (2007).

[16] J.-B. Shim, H.-W. Lee, S.-B. Lee, J. Yang, S. Moon, J.H. Lee, K. An, and S. W. Kim, J. Phys. Soc. Jpn. 76, 114005 (2007).

[17] S. C. Creagh, Phys. Rev. Lett. 98, 153901 (2007).

[18] J. D. Meiss, Rev. Mod. Phys. 64, 795 (1992).

[19] S. Wiggins, Chaotic Transport in Dynamical Systems (Springer-Verlag, New York, 1992).

[20] J. Yang, S. Moon, S.-B. Lee, J.-H. Lee, K. An, J.-B. Shim, H.-W. Lee, and S. W. Kim, Rev. Sci. Instrum. 77, 083103 (2006).

[21] J. U. Nöckel and A. D. Stone, Nature 385, 45 (1997).

[22] S. Kagami and I. Fukai, IEEE T. Microw. Theory 32, 455 (1984).

[23] J. Wiersig, J. Opt. A, Pure Appl. Opt. 5, 53 (2003).

[24] G. D. Birkhoff, Acta. Math. 50, 359 (1927).

[25] N. B. Rex, H. E. Tureci, H. G. L. Schwefel, R. K. Chang, and A. D. Stone, Phys. Rev. Lett. 88, 094102 (2002).

[26] S.-B. Lee, J.-H. Lee, J. S. Chang, H. J. Moon, S. W. Kim, and K. An, Phys. Rev. Lett. 88, 033903 (2002). 